\title{
SMOOTHING IN $C(X)$
}

ROBERT SINE ${ }^{1}$

1. In this note we prove convergence theorems for smoothing operators (conditional expectations) in the space of continuous functions. In this setting conditions which imply uniform convergence are found; indeed, in the form that the problem is set the conditions are necessary and sufficient. We give examples showing that many of the properties of conditional expectations on a measure space no longer hold in $C(X)$.

2. The space of all continuous real valued functions on a compact Hausdorff space $X$ is, as usual, $C(X)$. An operator $T$ on $C(X)$ is a Markov operator if $\|T\|=T 1=1$. The following is well known [1]; if the range of a Markov projection is a subalgebra of $C(X)$ the smoothing equation $T(f T g)=(T f)(T g)$ is satisfied and conversely if a Markov projection satisfies the smoothing equation then the range is an algebra. A subalgebra $A$ of $C(X)$ will be called smooth if there is a Markov projection onto $A$. The terminology is somewhat misleading; smoothness depends on the embedding of $A$ in $C(X)$ rather than intrinsic properties of $A$. Necessary and sufficient conditions for a subalgebra to be smooth are not known. However see Michael [3] for a sufficient condition and see Lloyd [2] for various examples. If we consider the map to constant functions defined by $f \rightarrow(\mu, f)$ where $\mu$ is any probability on $X$ we see that a smooth subalgebra may fail to have a unique smoothing projection.

Let $X$ consist of $[0,1] \cup\{1+1 / n: n \in N\} \cup\{-1 / n: n \in N\}$. Let $A_{1}$ be the $C(X)$ subalgebra of functions constant on $\{1+1 / n: n \in N\}$ $\cup\{-1\}$ and $A_{2}$ the functions constant on $\{-1 / n: n \in N\} \cup\{2\}$. Then $A_{1}$ and $A_{2}$ are both smooth subalgebras (and here we do have uniqueness of the smoothing operations) and $A_{1} \cap A_{2}$ fails to be smooth.

For the convergence theorems we will consider a nested sequence of smooth subalgebras, either increasing or decreasing. For the increasing sequence we define the final algebra $A_{\infty}=\cup A_{m}{ }^{-}$and in the decreasing case the final algebra is $A_{\infty}=\cap A_{n}$. Neither is in general smooth. For with $X$ as above we let $A_{n}$ be the subalgebra of $C(X)$ consisting of functions constant on the subset of $X$ contained in $[-1,2] \backslash(-1 / n, 1+1 / n)$. For an increasing nest we take $A_{n}$ to be

Received by the editors February 5, 1968.

1 This research was supported by the National Science Foundation. 
constant on $[-1 / n, 1+1 / n] \cap X$. In both cases the final algebras are not smooth.

3. If $P$ is a Markov projection there is a non-empty compact set $K$ of probabilities invariant under $T^{*}$ (Tychonov). Since uniqueness of projections in general fails even when existence is known we will assume that the projections commute.

LeMma. Let $A_{1} \subset A_{2}$ be two smooth subalgebras with given projections $P_{1}$ and $P_{2}$. Then $P_{1}$ and $P_{2}$ commute iff $K_{1} \subset K_{2}$.

Proof. We suppose first $P_{1} P_{2}=P_{2} P_{1}$. Let $\mu$ be in $K_{1}$. Then $\left(f, P_{2}^{*} \mu\right)$ $=\left(f, P_{2}^{*} P_{1}^{*} \mu\right)\left(f, P_{1}^{*} P_{2}^{*} \mu\right)=\left(P_{1} f, P_{2}^{*} \mu\right)=\left(P_{2} P_{1} f, \mu\right)=\left(P_{1} f, \mu\right)\left(f, P_{1}^{*} \mu\right)$ $=(f, \mu)$. So we have $P_{2} \mu=\mu$. Now conversely suppose $K_{1} \subset K_{2}$. Then $P_{1} P_{2} f(x)=\left(P_{1} P_{2} f, \varepsilon_{x}\right)=\left(P_{2} P_{1} f, P_{1}^{*} \varepsilon_{x}\right)=\left(f, P_{2}^{*} P_{1}^{*} \varepsilon_{x}\right)=\left(f, P_{1}^{*} \varepsilon_{x}\right)=$ $\left(P_{1} f, \varepsilon_{x}\right)=P_{1} f(x)=P_{2} P_{1} f(x)$.

Now if the projections commute the invariant sets $K_{n}$ are nested so we define $K_{\infty}=\bigcap K_{n}$ in the decreasing case and $K_{\infty}=U K_{n}-$ in the increasing case.

Theorem. Let $\left\{P_{n}\right\}$ be a commutative set of smoothing projections associated with a nested sequence of smooth subalgebras. Then $P_{n}$ converges in the strong operator topology iff $A_{\infty}$ separates the points of $K_{\infty}$. The operator defined by the limit is then a Markov smoothing projection onto $A_{\infty}$.

Proof. First we consider $A_{n} \downarrow A_{\infty}$. Let $\mathcal{E}(x)$ be the unit point mass at $x$ and let $\mu(x)$ be a $\omega^{*}$ cluster point of $\left\{P_{n}^{*} \mathcal{E}(x)\right\}$; since $P_{n}^{*} \mathcal{E}(x)$ is in $K_{n}$ we have $\mu(x)$ in $K_{\infty}$. Now if $f$ is in $A_{\infty}$ then $P_{n} f(x)$ is constant. So since $A_{\infty}$ separates the points of $K_{\infty}, P_{n}^{*} \mathcal{E}(x)$ has a unique cluster point so $P_{n}^{*} \varepsilon(x) \rightarrow \mu(x)$. The map $x \rightarrow \mu(x)$ is continuous in the topology of $K_{\infty}$ defined by the $A_{\infty}$ functions. However, this is just the $\omega^{*}$ topology restricted to $K_{\infty}$ (the $A_{\infty}$ topology is a weaker Hausdorf topology for the compact space $K_{\infty}$ ). By virtue of the $\omega^{*}$ continuity of $x \rightarrow \mu(x)$ we can define the (necessarily) Markov operator $P_{\infty} f(x)$ $=(f, \mu(x))$. We have $P_{n} \rightarrow P_{\infty}$ in the weak operator topology and wish to show uniform convergence of $P_{n} f$. Suppose $P_{\infty} g=0$ so $P_{n} g$ goes to zero pointwise. If $\left\|P_{n} g\right\|=r(n)>\delta>0$ we can always pick a probability $\mu(n)$ in $K_{n}$ so that $\left|\left(P_{n} g, \mu(n)\right)\right|=r(n)$. For this note that $P_{n} g$ is invariant under $P_{n}$. Pick $x$ so $\left|P_{n} g(x)\right|=r(n)$ and let $\mu(n)$ be the Cesaro limit of $\varepsilon(x)$. Now we can pass to a subnet so that $P_{\alpha} \mu(\alpha)$ $\rightarrow \mu(0)$ in $K_{\infty}$. But $\left|\left(g, P_{\alpha}^{*} \mu(\alpha)\right)\right| \rightarrow|(g, \mu(0))|=\left|\left(g, P_{\infty}^{*} \mu(0)\right)\right|=0$. Contradiction.

The increasing case is similar. We have $P_{n}^{*} \mathcal{E}(x)$ in $K_{n}$ so separation gives a unique cluster point and so convergence. Again continuity of 
$x \rightarrow \mu_{x}$ yields a weak operator limit $P_{\infty}$. Now if $P_{\infty} g=0$ and $\left\|P_{n} g\right\|$ $=r(n)>\delta>0$ we again pick a $P_{n}$-invariant probability $\mu(n)$ with $\left|\left(\mu(n), P_{n} g\right)\right|=r(n)$. Passage to a convergent subset again gives the contradiction.

If uniform convergence of $P_{n} f$ holds then we obtain a Markov idempotent with range an algebra $A_{\infty}$. If $\mu_{1}$ and $\mu_{2}$ are two $P_{\infty}$-invariant probabilities we pick $f$ to be any $C(X)$ function distinguishing $\mu_{1}$ and $\mu_{2}$. The function $P_{\infty} f$ is in $A_{\infty}$ and clearly still distinguishes $\mu_{1}$ and $\mu_{2}$.

\section{BIBLIOGRAPHY}

1. G. Birkoff, Moyennes de fonctions bornées. Algebre et theorie des nombres, Colloques Internationaux du Centre National de la Recherche Scientifique, no. 24, pp. 143-153, Centre National de la Recherche Scientifique, Paris, 1950.

2. S. P. Lloyd, On extreme averaging operators, Proc. Amer. Math. Soc. 14 (1963), 305-310.

3. E. Michael, $A$ linear transformation between function spaces, Proc. Amer. Math. Soc. 15 (1964), 407-409.

UNIVERSITY OF MinNESOTA 